\title{
ON THE POINTWISE CONVERGENCE OF THE EIGENFUNCTION EXPANSION ASSOCIATED WITH SOME ITERATED BOUNDARY VALUE PROBLEMS
}

\author{
JYOTI DAS AND PRABIR KUMAR SENGUPTA
}

\begin{abstract}
Given a boundary value problem consisting of a second-order differential equation and some boundary conditions, one can derive higher-order boundary value problems, called iterated boundary value problems, provided the coefficients in the second-order differential equation are sufficiently smooth. The problem of convergence of the eigenfunction expansions associated with boundary value problems of even order has been the central attraction for mathematicians since the beginning of this century. The idea of this paper is to single out some higher-order boundary value problems, for which the question of convergence of the said expansion is completely answered by the similar problem associated with the second-order boundary value problem responsible for the generation of the iterated boundary value problem.
\end{abstract}

1. Introduction. The problem of the convergence, pointwise or in respect to some norm, of the eigenfunction expansion associated with some boundary value problems has attracted the attention of many mathematicians since the beginning of this century. In 1910, Hermann Weyl [14] first proved that every second-order differential equation

$$
L[y] \equiv-\left(p y^{(1)}\right)^{(1)}+q y=\lambda y, \quad 0 \leq x<\infty
$$

(where $\lambda=\mu+i \nu, \nu \neq 0, p, q$ are real-valued functions on $[0, \infty), p>0, y^{(r)}=$ $\left.d^{r} / d x^{r}, r=1,2\right)$, has at least one solution belonging to the class $\mathcal{L}^{2}=\mathcal{L}^{2}[0, \infty)$ of all Lebesgue square-integrable functions on $[0, \infty)$. This initiated the study of the convergence problem in the $\mathcal{L}^{2}$-space setting and it has been proved that the eigenfunction expansion of any $f \in \mathcal{L}^{2}[0, \infty)$ associated with the boundary value problem

$$
\left\{\begin{array}{l}
L[y]=\lambda y, \quad \lambda=\mu+i \nu, 0 \leq x<\infty, \\
y(0) \cos \alpha+y^{(1)}(0) \sin \alpha=0 \quad(0 \leq \alpha<\pi), \\
y \in \mathcal{L}^{2}[0, \infty),
\end{array}\right.
$$

converges to $f$ in the normed linear space $\mathcal{L}^{2}[0, \infty)$. The problem becomes intricate when one considers the pointwise convergence of the said expansion. Titchmarsh [13] has developed a theory to prove the convergence of the eigenfunction expansion corresponding to a given function $f \in \mathcal{L}^{2}$ at each point $x \in[0, \infty)$, whenever the Fourier series of $f$ converges to $f$ at that point, under suitable restrictions on $p$ and $q$ or on $f$.

Received by the editors June 2, 1983 and, in revised form, March 5, 1985.

1980 Mathematics Subject Classification. Primary 34B25.

Key words and phrases. Bilinear concomitant, boundary condition function, boundary value problem, convergence under Fourier conditions, Hilbert space, Lebesgue square-integrable solution, limit-point case at infinity, limit- $n$ case at infinity, resolvent operator, simple closed contour. 
For suitable $p, q$, formal powers $L^{n}[\cdot]$, where the integer $n \geq 1$, of $L[\cdot]$ may be obtained according to the process of iteration

$$
L^{n}[y]=L\left[L^{n-1}[y]\right] \quad(n \geq 1),
$$

with the convention $L^{0}[y]=y$. Then a $(2 n)$ th order iterated boundary value problem $\mathrm{I}_{n}$ may be defined as follows $(n \geq 2)$ :

$$
\left\{\begin{array}{l}
L^{n}[y]=\lambda^{n} y \\
B(y)=B_{1}(y)=\cdots=B_{n-1}(y)=0
\end{array}\right.
$$

where $B_{r}(y) \equiv B\left(L^{r}[y]\right)$ and $B(y) \equiv y(0) \cos \alpha+y^{(1)}(0) \sin \alpha(0 \leq \alpha<\pi)$.

It is natural that anyone will be interested in the investigation of the problem of convergence of the eigenfunction expansion associated with the iterated boundary value problem $\left(\mathrm{I}_{n}\right)$. The idea of this paper is to reveal that the pointwise convergence of the eigenfunction expansion of any $f \in \mathcal{L}^{2}[0, \infty)$ associated with the iterated boundary value problem $\left(\mathrm{I}_{n}\right)$ can very easily be derived from the corresponding problem associated with (I) under those restrictions on $p, q$ which are sufficient for the formation of $L^{n}[y](n \geq 1)$, and are such that only $n$ linearly independent solutions of $L^{n}[y]=\lambda^{n} y(\lambda=\mu+i \nu, \nu \neq 0)$ belong to $\mathcal{L}^{2}[0, \infty)$ (in short, we say that $L^{n}[\cdot]$ is in the limit- $n$ condition at infinity). The result thus obtained is stated in the following theorem.

THEOREM. Let

(a) $L[\cdot]$ be in the limit-point condition at infinity (i.e. $L[y]=\lambda y, \operatorname{im} \lambda \neq 0$, has only one linearly independent solution belonging to $\left.\mathcal{L}^{2}[0, \infty)\right)$;

(b) the coefficients $p, q$ are real-valued functions on $[0, \infty)$ and for some integer $n$ with $n \geq 2$,

(i) let $\bar{p}^{(2 n-2)}$ be absolutely continuous on $[0, X]$ for all $X>0$ and let $p(x)>0$ for all $x \in[0, \infty)$,

(ii) let $q^{(2 n-3)}$ be absolutely continuous on $[0, X]$ for all $X>0$ (see Everitt-Giertz [6] for these conditions on $p, q$ ),

\section{(c) $L^{n}[\cdot](n \geq 2)$ be in the limit-n condition at infinity.}

Then the eigenfunction expansion of an arbitrary function $f \in \mathcal{L}^{2}[0, \infty)$ associated with the boundary value problem $\left(\mathrm{I}_{n}\right)(n \geq 2)$ converges to $f(x)$ at any point $x \in[0, \infty)$, provided the eigenfunction expansion of $f$ corresponding to the boundary value problem (I) at that point $x$ converges to $f(x)$.

Some useful results regarding the boundary value problem (I) are given in $\S 2$. In $\S 3$ we present the proof of this theorem for the case $n=2$. $\S 4$ gives a sketch of the proof of the theorem for the case $n>2$, details being omitted for want of space. To clarify the result, an example is dealt with in $\S 5$.

2. Some useful results. For the boundary value problem (I) given by

$$
\left\{\begin{array}{l}
L[y]=-\left(p y^{(1)}\right)^{(1)}+q y=\lambda y, \quad \lambda=\mu+i \nu, 0 \leq x<\infty \\
B(y) \equiv y(0) \cos \alpha+y^{(1)}(0) \sin \alpha=0 \quad(0 \leq \alpha<\pi) \\
y \in \mathcal{L}^{2}[0, \infty)
\end{array}\right.
$$

let $\phi(\cdot, \lambda)$ denote the boundary condition function as defined by Kodaira [7]. As the bilinear concomitant associated with the differential expression $L[\cdot]$ is

$$
[u v]_{2}=p(x)\left[u(x) \bar{v}^{(1)}(x)-u^{(1)}(x) \bar{v}(x)\right]
$$


it is easy to verify that the boundary condition function $\phi$ is required to satisfy the following $(0 \leq \alpha<\pi)$ :

$$
p(0) \phi(0, \lambda)=-\sin \alpha, \quad p(0) \phi^{(1)}(0, \lambda)=\cos \alpha .
$$

Let $\theta=\theta(x, \lambda)$ be that solution of $L[y]=\lambda y$ which satisfies the initial conditions

$$
\theta(0, \lambda)=-\cos \alpha, \quad \theta^{(1)}(0, \lambda)=-\sin \alpha .
$$

It can be easily verified that

$$
[\phi \theta]_{2}=1 .
$$

H. Weyl [14] has proved that there exists an analytic function $m(\lambda)$, such that, if $\operatorname{im} \lambda \neq 0$, then

$$
\psi(x, \lambda)=\theta(x, \lambda)+m(\lambda) \phi(x, \lambda) \in \mathcal{L}^{2} .
$$

For any $f \in \mathcal{L}^{2}$, Titchmarsh $[13]$ defined the function $\Phi \equiv \Phi(\cdot, \lambda)=\Phi(\cdot, \lambda ; f)$ known as the resolvent operator associated with the BVP (I) as follows:

$$
\begin{aligned}
\Phi(x, \lambda) & =\Phi(x, \lambda ; f) \\
& =\Psi(x, \lambda) \int_{0}^{x} \phi(t, \lambda) f(t) d t+\phi(x, \lambda) \int_{x}^{\infty} \psi(t, \lambda) f(t) d t .
\end{aligned}
$$

In Chapter III of [13], Titchmarsh proved that the eigenfunction expansion of any given function $f \in \mathcal{L}^{2}$ at a given point $x$ will converge to $f(x)$ if and only if

$$
f(x)=\lim _{R \rightarrow \infty} \lim _{\delta \rightarrow 0+}\left\{\frac{i}{\pi} \int_{-R+i \delta}^{R+i \delta} \Phi(x, \lambda) d \lambda\right\} .
$$

Many sets of conditions on $p$ and $q$ or on $f$ or on all of them have been stipulated to ensure the validity of (2.7) (see [8]-[12]).

Our aim here is to show that the convergence problem with respect to any iterated boundary value problem $\left(\mathrm{I}_{n}\right)$ can be related to the convergence problem with respect to (I).

3. The case $n=2$. The BVP $\left(\mathrm{I}_{2}\right)$ is exhibited below:

$$
\begin{aligned}
& L^{2}[y]=\lambda^{2} y, \quad \lambda=\mu+i \nu, 0 \leq x<\infty, \\
& B(y)=B(L[y])=0,
\end{aligned}
$$

where $B(y) \equiv y(0) \cos \alpha+y^{(1)}(0) \sin \alpha,(0 \leq \alpha<\pi)$.

The bilinear concomitant $[u v]_{4}$ associated with the differential equation of $\left(\mathrm{I}_{2}\right)$ is as follows:

$$
\begin{aligned}
{[u v]_{4} \equiv } & p^{2}(x)\left\{u^{(3)}(x) \bar{v}(x)-u^{(2)}(x) \bar{v}^{(1)}(x)+u^{(1)}(x) \bar{v}^{(2)}(x)-u(x) \bar{v}^{(3)}(x)\right\} \\
& +2 p(x) p^{(1)}(x)\left\{u^{(2)}(x) \bar{v}(x)-u(x) \bar{v}^{(2)}(x)\right\} \\
& +\left\{2 p(x) q(x)-p(x) p^{(2)}(x)\right\}\left\{u(x) \bar{v}^{(1)}(x)-u^{(1)}(x) \bar{v}(x)\right\}
\end{aligned}
$$

The boundary condition functions $\phi_{r}\left(\cdot, \lambda^{2}\right)(r=1,2)[4,5]$ are then required to satisfy

$$
\left\{\begin{array}{l}
\phi_{1}(0, \lambda)=\phi_{1}^{(1)}\left(0, \lambda^{2}\right)=0 \\
p^{2}(0) \phi_{1}^{(2)}\left(0, \lambda^{2}\right)=\sin \alpha \\
p^{3}(0) \phi_{1}^{(3)}\left(0, \lambda^{2}\right)=-\left[p(0) \cos \alpha+p^{(1)}(0) \sin \alpha\right]
\end{array}\right.
$$


and

$$
\left\{\begin{array}{l}
p(0) \phi_{2}\left(0, \lambda^{2}\right)=-\sin \alpha, \quad p(0) \phi_{2}^{(1)}\left(0, \lambda^{2}\right)=\cos \alpha \\
p^{2}(0) \phi_{2}^{(2)}\left(0, \lambda^{2}\right)=-\left[q(0) \sin \alpha+p^{(1)}(0) \cos \alpha\right] \\
p^{3}(0) \phi_{2}^{(3)}\left(0, \lambda^{2}\right)=\left\{2 p^{(1)}(0) q(0)-p(0) q^{(1)}(0)\right\} \sin \alpha \\
\quad+\left\{2\left[p^{(1)}(0)\right]^{2}+p(0) q(0)-p(0) p^{(2)}(0)\right\} \cos \alpha
\end{array}\right.
$$

If 1 and $u$ denote the two distinct roots of unity, it can be easily verified that each of $\phi(\cdot, \lambda), \phi(\cdot, \lambda u), \theta(\cdot, \lambda)$ and $\theta(\cdot, \lambda u)$ satisfies the differential equation $L^{2}[y]=\lambda^{2} y$ of $\left(\mathrm{I}_{2}\right)$, and they are linearly independent if $\lambda \neq 0$. This allows us to express the boundary condition functions $\phi_{r}\left(\cdot, \lambda^{2}\right)(r=1,2)$ in terms of these four functions. In fact, we have,

$$
\begin{aligned}
& \phi_{1}\left(x, \lambda^{2}\right)=\frac{1}{2 \lambda}[\phi(x, \lambda)-\phi(x, \lambda u)], \\
& \phi_{2}\left(x, \lambda^{2}\right)=\frac{1}{2}[\phi(x, \lambda)+\phi(x, \lambda u)] .
\end{aligned}
$$

If we choose the functions $\theta_{r}\left(\cdot, \lambda^{2}\right)(r=1,2)$ as

$$
\begin{aligned}
& \theta_{1}\left(x, \lambda^{2}\right)=\frac{1}{2}[\theta(x, \lambda)+\theta(x, \lambda u)], \\
& \theta_{2}\left(x, \lambda^{2}\right)=\frac{1}{2 \lambda}[\theta(x, \lambda)-\theta(x, \lambda u)],
\end{aligned}
$$

it can be easily verified that ( $\delta_{r s}$ denoting Kronecker's delta)

$$
\left[\phi_{r} \theta_{s}\right]_{4}=\delta_{r s} \quad(r, s=1,2), \quad\left[\theta_{1} \theta_{2}\right]_{4}=0, \quad\left[\phi_{1} \phi_{2}\right]=0 .
$$

According to Everitt $[5, \S 18]$, there exist analytic functions $m_{r s}(\cdot)(r, s=1,2)$ so that, for $\operatorname{im} \lambda \neq 0$,

$$
\psi_{r}\left(x, \lambda^{2}\right)=\theta_{r}\left(x, \lambda^{2}\right)+\sum_{s=1}^{2} m_{r s} \phi_{s}\left(x, \lambda^{2}\right) \in \mathcal{L}^{2} .
$$

On the other hand $\psi(\cdot, \lambda)$ and $\psi(\cdot, \lambda u)$ are two linearly independent (for $\operatorname{im} \lambda \neq 0$ ) square-integrable solutions of $L^{2}[y]=\lambda^{2} y$. Since, according to hypothesis (c), $L^{2}[\cdot]$ is in the limit-2 condition, the two square-integrable solutions $\psi_{r}\left(\cdot, \lambda^{2}\right)(r=1,2)$ of $L^{2}[y]=\lambda^{2} y$ must be a linear combination of the solutions $\psi(\cdot, \lambda)$ and $\psi(\cdot, \lambda u)$, say

$$
\psi_{r}\left(x, \lambda^{2}\right)=A_{r} \psi(x, \lambda)+B_{r} \psi(x, \lambda u) \quad(r=1,2) .
$$

It has been proved in [4] that there is a relation between the two bilinear forms given in (2.1) and (3.1) which may be exhibited as follows:

$$
[u v]_{4}=[L[u] v]_{2}+[u L[v]]_{2} \text {. }
$$

From (3.8) we deduce that $\left[\phi_{r} \psi_{s}\right]_{4}=\delta_{r s}(r, s=1,2)$. Using this result and the expressions for $\phi_{1}, \phi_{2}$ given in (3.4) and (3.5) we can easily derive from (3.10) that

$$
\begin{aligned}
& \psi_{1}\left(x, \lambda^{2}\right)=\frac{1}{2}[\psi(x, \lambda)+\psi(x, \lambda u)] \\
& \psi_{2}\left(x, \lambda^{2}\right)=\frac{1}{2 \lambda}[\psi(x, \lambda)-\psi(x, \lambda u)]
\end{aligned}
$$


Chaudhuri and Everitt [3] have proved that the resolvent operator $\Phi_{2}=\Phi_{2}\left(x, \lambda^{2}\right)=$ $\Phi_{2}\left(x, \lambda^{2} ; f\right)$ associated with the BVP $\left(\mathrm{I}_{2}\right)$ is given by

$$
\begin{aligned}
\Phi_{2}\left(x, \lambda^{2}\right) & =\Phi_{2}\left(x, \lambda^{2} ; f\right) \\
& =\sum_{r=1}^{2}\left\{\psi_{r}\left(x, \lambda^{2}\right) \int_{0}^{x} \phi_{r}\left(t, \lambda^{2}\right) f(t) d t+\phi_{r}\left(x, \lambda^{2}\right) \int_{x}^{\infty} \psi_{r}\left(t, \lambda^{2}\right) f(t) d t\right\} .
\end{aligned}
$$

It is now a matter of simple substitution of the expressions for $\phi_{r}$ and $\psi_{r}$ as given in (3.4), (3.5), (3.12) and (3.13), in the expression (3.14) of $\Phi_{2}\left(x, \lambda^{2}\right)$ to deduce that

$$
\Phi_{2}\left(x, \lambda^{2} ; f\right)=\frac{1}{2 \lambda}[\Phi(x, \lambda ; f)+u \Phi(x, \lambda u ; f)] .
$$

This is the key result of our exposition. Let $\Gamma_{1}$ be a simple closed curve in the $\lambda$-plane, symmetric about the real $\lambda$-axis. Let the image of the upper half of $\Gamma_{1}$ into the $\lambda^{2}$-plane be $\Gamma_{2}$, which is clearly a simple closed curve. Further, as one moves round the closed contour $\Gamma_{1}$ once, one has to move round $\Gamma_{2}$ twice. Hence

$$
\begin{aligned}
2 \int_{\Gamma_{2}} \Phi_{2}\left(x, \lambda^{2} ; f\right) d\left(\lambda^{2}\right) & =\int_{\Gamma_{1}} \frac{1}{2 \lambda}[\Phi(x, \lambda ; f)+u \Phi(x, \lambda u ; f)] d\left(\lambda^{2}\right) \\
& =\int_{\Gamma_{1}} \Phi(x, \lambda ; f) d \lambda+\int_{\Gamma_{1}} \Phi(x, \lambda u ; f) d(\lambda u) .
\end{aligned}
$$

One now requires only routine arguments to establish that if the eigenfunction expansion associated with the BVP (I) converges, then so does the eigenfunction expansion associated with the boundary value problem $\left(I_{2}\right)[1,2]$.

4. The case $n>2$. In the case of the $(2 n)$ th order boundary value problem $\left(\mathrm{I}_{n}\right)$, we adopt a different approach. The hypothesis (c) that $L^{n}[\cdot]$ is in the limit- $n$ case at infinity, enables one to express the $n$ square-integrable solutions $\chi_{r}\left(x, \lambda^{n}\right)(r=$ $1,2, \ldots, n)$ of $L^{n}[y]=\lambda^{n} y$ in terms of the $n$ functions $\psi\left(x, \lambda v^{r}\right)(r=0,1,2, \ldots$, $n-1)$, where $1, v, v^{2}, \ldots, v^{n-1}$ are the $n$ distinct $n$th roots of unity, as

$$
\chi_{r}\left(x, \lambda^{n}\right)=\sum_{s=0}^{n-1} A_{r s} \psi\left(x, \lambda v^{s}\right) \quad(r=1,2, \ldots, n)
$$

but it becomes a tiresome job to determine the coefficients $A_{r s}$. Instead we prove that the function $\Phi_{n}\left(=\Phi_{n}\left(x, \lambda^{n}\right)=\Phi_{n}\left(x, \lambda^{n} ; f\right)\right)$ defined by

$$
\begin{aligned}
\Phi_{n}\left(x, \lambda^{n}\right) & =\Phi_{n}\left(x, \lambda^{n} ; f\right) \\
& =\frac{1}{n \lambda^{n-1}}\left[\Phi(x, \lambda ; f)+v \Phi(x, \lambda v ; f)+\cdots+v^{n-1} \Phi\left(x, \lambda v^{n-1}, f\right)\right]
\end{aligned}
$$

satisfies all the requisite conditions in order to be the resolvent operator of the BVP $\left(\mathrm{I}_{n}\right)$, viz.

(a) $L^{n}\left[\Phi_{n}\right]=\lambda^{n} \Phi_{n}+f$ for all $f \in \mathcal{L}^{2}$, and

(b) $B\left(L^{k}\left[\Phi_{n}\right]\right)=0(k=0,1,2, \ldots, n-1)$. 
For the proof of this we first note that

$$
\begin{aligned}
L\left[\Phi_{n}\left(x, \lambda^{n} ; f\right)\right]= & \frac{1}{n \lambda^{n-1}}[\{\lambda \Phi(x, \lambda ; f)+f\}+v\{\lambda v \Phi(x, \lambda v, f)+f\} \\
& \left.+\cdots+v^{n-1}\left\{\lambda v^{n-1} \Phi\left(x, \lambda v^{n-1}, f\right)+f\right\}\right] \\
= & \frac{1}{n \lambda^{n-2}}\left[\Phi(x, \lambda, f)+v^{2} \Phi(x, \lambda v, f)+\cdots+v^{2(n-1)} \Phi\left(x, \lambda v^{n-1}, f\right)\right]
\end{aligned}
$$

since $1+v+v^{2}+\cdots+v^{n-1}=0, v$ being an $n$th root of unity. Further, for any positive integer $k$,

$$
\left(1-v^{k}\right)\left(1+v^{k}+v^{2 k}+\cdots+v^{k(n-1)}\right)=1-v^{n k}=0
$$

and so it follows that $1+v^{k}+v^{2 k}+\cdots+v^{k(n-1)}=0$ if $v^{k} \neq 1$. Using this we can prove from (4.2) by iteration that, for $k=1,2, \ldots, n-1$,

$$
\begin{aligned}
L^{k}\left[\Phi_{n}\left(x, \lambda^{n}, f\right)\right]=\frac{1}{n \lambda^{n-k-1}}[\Phi( & x, \lambda, f)+v^{k+1} \Phi(x, \lambda v, f) \\
& \left.+\cdots+v^{(k+1)(n-1)} \Phi\left(x, \lambda v^{n-1}, f\right)\right] .
\end{aligned}
$$

Hence

$$
L^{n-1}\left[\Phi_{n}\left(x, \lambda^{n}, f\right)\right]=\frac{1}{n}\left[\Phi(x, \lambda ; f)+\Phi(x, \lambda v, f)+\cdots+\Phi\left(x, \lambda v^{n-1}, f\right)\right],
$$

noting that $v^{n}=1$. Operating with $L$ once again we get

$$
\begin{aligned}
L^{n}\left[\Phi_{n}\left(x, \lambda^{n}, f\right)\right]= & \frac{1}{n}[\{\lambda \Phi(x, \lambda, f)+f\}+\{\lambda v \Phi(x, \lambda v, f)+f\} \\
& \left.+\cdots+\left\{\lambda v^{n-1} \Phi\left(x, \lambda v^{n-1}, f\right)+f\right\}\right] \\
& =\frac{\lambda}{n}\left[\Phi(x, \lambda ; f)+v \Phi(x, \lambda v, f)+\cdots+v^{n-1} \Phi\left(x, \lambda v^{n-1}, f\right)\right]+f \\
& =\frac{\lambda}{n} \cdot n \lambda^{n-1} \Phi_{n}\left(x, \lambda^{n}, f\right)+f \quad(\text { from }(4.1)) \\
& =\lambda^{n} \Phi_{n}\left(x, \lambda^{n}, f\right)+f .
\end{aligned}
$$

This proves (a).

Noting that $B$ operates linearly, we get

$$
\begin{array}{rlrl}
B\left(L^{k}\left[\Phi_{n}\right]\right)= & \frac{1}{n \lambda^{n-k-1}}[ & B(\Phi(x, \lambda, f))+v^{k+1} B(\Phi(x, \lambda v, f)) \\
& \left.+\cdots+v^{(k+1)(n-1)} B\left(\Phi\left(x, \lambda v^{n-1}, f\right)\right)\right] \\
= & 0,
\end{array}
$$

since $B(\Phi(x, \lambda, f))=0$ for all $\lambda$ as the initial values of $\Phi(\cdot, \lambda)$ are independent of $\lambda$. This completes the proof of (b). The rest of the proof of the theorem can now be completed as in the previous section.

5. An example: $p \equiv 1, q \equiv 0, \alpha=0$. In this case, the BVP (I) becomes

$$
\left\{\begin{array}{l}
L_{0}[y] \equiv-y^{(2)}=\lambda y \quad(\lambda=\mu+i \nu) \\
B(y) \equiv y(0)=0
\end{array}\right.
$$


If $\lambda=s^{2}, 0<\arg \lambda<\pi, \operatorname{im} s>0$, the two solutions of $-y^{(2)}=\lambda y$ are $e^{i s x}$ and $e^{-i s x}$, of which $e^{i s x} \in \mathcal{L}^{2}$ but $e^{-i s x} \notin \mathcal{L}^{2}$. For suitable $f \in \mathcal{L}^{2}$, the corresponding eigenfunction expansion assumes the form:

$\left(\mathrm{A}_{1}\right)$

$$
\frac{-1}{2 \pi i} \int_{-\infty}^{\infty} \frac{1}{s}\left(e^{i s x}-e^{-i s x}\right) d\left[\int_{0}^{\infty}\left\{\frac{s}{y}\left(e^{i s y}+e^{-i s y}\right)+\frac{i}{y^{2}}\left(e^{i s y}-e^{-i s y}\right)\right\} f(y) d y\right]
$$

and that corresponding to the $\mathrm{BVP}\left(\mathrm{I}^{\prime}\right)$, obtained by replacing $L_{0}[y]=\lambda y$ by $L_{0}[y]=-\lambda y$ is

$$
\left(\mathrm{A}_{2}\right) \frac{-1}{2 \pi i} \int_{-\infty}^{\infty} \frac{1}{s}\left(e^{s x}-e^{-s x}\right) d\left[\int_{0}^{\infty}\left\{\frac{s}{y}\left(e^{s y}+e^{-s y}\right)-\frac{1}{y^{2}}\left(e^{s y}-e^{-s y}\right)\right\} f(y) d y\right] \text {. }
$$

Now, the squared BVP (II) of (I) or (I') consists of

$$
\left\{\begin{array}{l}
L^{2}[y] \equiv y^{(4)}=\lambda^{2} y \\
B(y) \equiv y(0)=0 \\
B(L[y]) \equiv y^{(2)}(0)=0
\end{array}\right.
$$

After some tedious calculations one can prove that the expansion of suitable $f \in \mathcal{L}^{2}$ associated with the BVP (II) becomes (see [1, Chapter III, p. 49]

$$
\begin{aligned}
& \frac{-1}{4 \pi} \int_{-\infty}^{\infty} s^{-3}\left[e^{s x}-e^{-s x}+i e^{i s x}-i e^{-i s x}\right] \\
& \quad \times d\left[\int _ { 0 } ^ { \infty } \left\{\left(\frac{6 i}{y^{4}}+\frac{6 s}{y^{3}}-\frac{3 i s^{2}}{y^{2}}-\frac{s^{3}}{y}\right) e^{i s y}\right.\right. \\
& \left.\left.\quad+\left(\frac{-6 i}{y^{4}}+\frac{6 s}{y^{3}}+\frac{3 i s^{2}}{y^{2}}-\frac{s^{3}}{y}\right) e^{-i s y}\right\} f(y) d y\right] \\
& +\frac{-1}{4 \pi} \int_{-\infty}^{\infty} s^{-2}\left[e^{s x}-e^{-s x}-i e^{i s x}+i e^{-i s x}\right] \\
& \quad \times d\left[\int_{0}^{\infty}\left\{\frac{s}{y}\left(e^{i s y}+e^{-i s y}\right)-\frac{i}{y^{2}}\left(e^{i s y}-e^{-i s y}\right)\right\} f(y) d y\right] .
\end{aligned}
$$

It is therefore clear that the convergence of $\left(A_{1}\right)$ implies that of $\left(A_{2}\right)$ and hence the convergence of (B) follows.

The mere appearance of $(\mathrm{B})$ gives one the idea of how complicated the expansion of an arbitrary function in $\mathcal{L}^{2}$ corresponding to a fourth-order BVP is, not to speak of the task of proving its convergence. The present paper singles out a class of higher even order BVPs for which the convergence of the corresponding expansion can be deduced from sole knowledge of the corresponding second-order BVP.

ACKNOWLEDGEMENT. The authors are thankful to the referee for his valuable suggestions.

\section{REFERENCES}

1. Chaudhuri Jyoti, Some problems in the theory of eigenfunction expansions, Thesis, Oxford Univ., 1964.

2. - On the convergence of the eigenfunction expansion associated with a fourth-order differential equation, Quart. J. Math. Oxford Ser. (2) 15 (1964), 258-274.

3. Chaudhuri Jyoti and W. N. Everitt, On an eigenfunction expansion associated with a fourth-order differential equation, Quart. J. Math. Oxford Ser. (2) 20 (1969), 195-213. 
4. __ On the square of a formally selfadjoint differential expression, J. London Math. Soc. (2) 1 (1969), 661-673.

5. W. N. Everitt, Fourth-order singular differential equations, Math. Ann. 149 (1963), 320340.

6. W. N. Everitt and M. Giertz, On some properties of powers of a formally selfadjoint differential expression, Proc. London Math. Soc. (3) 24 (1972), 149-170.

7. K. Kodaira, On ordinary differential equations of any even order and the corresponding eigenfunction expansions, Amer. J. Math. 72 (1950) , 502-544.

8. J. B. Mcleod, Three problems in eigenfunction expansions, Thesis, Oxford Univ., 1958.

9. - Convergence of eigenfunction expansions under Fourier conditions. I, Proc. London Math. Soc. (3) 12 (1962), 144-166.

10. D. B. Sears, An expansion in eigenfunctions, Proc. London Math. Soc. 53 (1951), 396-421.

11. E. C. Titchmarsh, On the convergence of eigenfunction expansions, Quart. J. Math. Oxford 3 (1952), 139-144.

12. _ On the convergence of eigenfunction expansions. II, Quart. J. Math. Oxford Ser. (2) 8 (1957), 236-240.

13. I, 2nd ed., Oxford Univ. Press, London, 1962.

14. H. Weyl, Über gewohnliche Differential gleichungen mit Singularitaten und die zugehorigen Entwicklungen, Math. Ann. 68 (1910), 220-269.

Department of Pure Mathematics, Calcutta University, Calcutta 700019 , INDIA 